[RAdiocarbon, Vol. 12, No. 2, 1970, P. 496-502]

\title{
GLASGOW UNIVERSITY RADIOCARBON MEASUREMENTS III
}

\author{
M. S. BAXTER* and A. WALTON
}

Chemistry Department, The University, Glasgow, W.2

\section{INTRODUCTION}

The following list presents results obtained during 1968-69 on a series of samples chosen to investigate temporal variations of $\mathrm{C}^{14}$ concentrations in the atmosphere during the past century. Together with data presented previously (Radiocarbon, 1969, v. 11, p. 45-52) they constitute a study of annual variations of $\mathrm{C}^{14}$ activities at $\mathrm{N}$ temperate latitudes.

Procedures for the analysis of a variety of organic and inorganic materials were previously reported and these have remained virtually unchanged. In some instances $\mathrm{C}^{14}$ concentrations were revised slightly in view of mass spectrometric analyses for $\mathrm{C}^{13} / \mathrm{C}^{12}$ ratios. All $\delta \mathrm{C}^{14}$ and $\Delta$ values of recent samples are decay-corrected, although this correction is very small.

\section{ACKNOWLEDGMENTS}

One of us (M.S.B.) gratefully acknowledges the financial support of a Science Research Council Studentship, during the tenure of which this work was performed.

\section{SPIRIT SAMPLES}

The study of atmospheric $\mathrm{C}^{14}$ concentrations in past years through analyses of malt whiskies of known age has continued. Results pub. in Radiocarbon, 1969, v. 11, p. 43-52 established the reliability of malt whiskies as indicators of atmospheric $\mathrm{C}^{14}$ concentrations during barley growth periods.

Malt whisky, Scotland series

\begin{tabular}{cccccc}
$\begin{array}{c}\text { Sample } \\
\text { no. }\end{array}$ & $\begin{array}{c}\text { Barley } \\
\text { coll. } \\
\text { date }\end{array}$ & $\begin{array}{c}\text { Distill. } \\
\text { date }\end{array}$ & $\delta \mathrm{C}^{14 \%} \%$ & $\delta \mathrm{C}^{13 \%} \%$ & $\Delta \%$ \\
\hline GU-228 & 1919 & 1920 & $-1.6 \pm 0.6$ & -27.1 & $-1.2 \pm 0.6$ \\
GU-229 & 1920 & 1921 & $-2.0 \pm 0.6$ & -25.7 & $-1.8 \pm 0.6$ \\
GU-230 & 1925 & 1926 & $-3.4 \pm 0.6$ & -27.7 & $-2.8 \pm 0.6$ \\
GU-231 & 1935 & 1936 & $-1.7 \pm 0.5$ & -27.6 & $-1.2 \pm 0.5$ \\
GU-232 & 1939 & 1940 & $-3.5 \pm 0.6$ & -28.1 & $-2.9 \pm 0.6$ \\
GU-233 & 1947 & 1948 & $-5.7 \pm 0.5$ & -27.9 & $-5.2 \pm 0.5$ \\
GU-234 & 1947 & 1948 & $-5.9 \pm 0.7$ & -29.1 & $-5.1 \pm 0.8$
\end{tabular}

II. VINTAGE WINE SAMPLES

L'Orange and Zimen (1968) have shown that a good correlation exists between atmospheric $\mathrm{C}^{14}$ concentrations and those in vintage wine

* Department of Earth and Space Sciences, State University of New York, Stony Brook, Long Island, New York. 
samples. To extend our knowledge of past atmospheric $\mathrm{C}^{14}$ concentrations a number of French and Portuguese wines were analyzed.

\begin{tabular}{clcccc}
$\begin{array}{c}\text { Sample } \\
\text { no. }\end{array}$ & $\begin{array}{c}\text { Sample } \\
\text { site }\end{array}$ & $\mathrm{Yr}$ & $\delta \mathrm{C}^{14 \%} \%$ & $\delta \mathrm{C}^{13 \%} \%$ & $\Delta \%$ \\
\hline GU-238 & Portugal & 1897 & $-1.7 \pm 0.5$ & -30.2 & $-0.7 \pm 0.5$ \\
GU-239 & France & 1906 & $-2.8 \pm 0.6$ & -29.2 & $-2.0 \pm 0.6$ \\
GU-240 & France & 1907 & $-4.7 \pm 0.5$ & -38.3 & $-2.2 \pm 0.5$ \\
GU-241 & France & 1907 & $-2.7 \pm 0.5$ & -27.8 & $-2.2 \pm 0.5$ \\
GU-242 & France & 1908 & $-3.3 \pm 0.5$ & -29.9 & $-2.4 \pm 0.5$ \\
GU-243 & France & 1914 & $-2.4 \pm 0.5$ & -30.5 & $-1.3 \pm 0.5$ \\
GU-244 & France & 1914 & $-1.0 \pm 1.1$ & -29.4 & $-0.1 \pm 1.1$ \\
GU-245 & Portugal & 1917 & $-4.0 \pm 0.6$ & -31.6 & $-2.8 \pm 0.6$ \\
GU-246 & France & 1918 & $+97.6 \pm 1.7$ & -29.6 & $+99.4 \pm 1.8$
\end{tabular}

Comments: high $\Delta$ value indicative of 1963 sample. Since lab contamination of sample to such an extent would seem impossible, discrepancy appears due to mistaken identity of sample. The analysis, however, reveals the possibility of applying $\mathrm{C}^{14}$ analysis to dating of recent wines even though accuracy of age-assessment may be limited within certain time periods.

\begin{tabular}{clcccc}
$\begin{array}{c}\text { Sample } \\
\text { no. }\end{array}$ & $\begin{array}{c}\text { Sample } \\
\text { site }\end{array}$ & Yr & $\delta \mathrm{C}^{14 \%}$ & $\delta \mathrm{C}^{13 \%} \%$ & $\Delta \%$ \\
\hline GU-247 & France & 1920 & $-1.6 \pm 0.5$ & -30.5 & $-0.5 \pm 0.5$ \\
GU-248 & France & 1926 & $-3.3 \pm 0.6$ & -28.7 & $-2.5 \pm 0.6$ \\
GU-249 & Portugal & 1927 & $-4.6 \pm 0.5$ & -31.6 & $-3.3 \pm 0.5$ \\
GU-250 & France & 1928 & $-3.2 \pm 0.6$ & -32.0 & $-1.8 \pm 0.6$ \\
GU-251 & France & 1928 & $-2.2 \pm 0.5$ & -28.4 & $-1.5 \pm 0.5$ \\
GU-252 & France & 1929 & $-2.2 \pm 0.5$ & -31.7 & $-1.0 \pm 0.6$ \\
GU-253 & France & 1929 & $-2.8 \pm 0.5$ & -31.4 & $-1.6 \pm 0.5$ \\
GU-254 & Portugal & 1929 & $-2.1 \pm 0.6$ & -29.8 & $-1.2 \pm 0.6$
\end{tabular}

III. TREE SEED SAMPLES

In a study of atmospheric $\mathrm{C}^{14}$ concentrations during the period 19591968 a number of tree seeds (subm. and id. by U. K. Forestry Comm.) have been analyzed. The seeds, stored in vacuum since collection, represent a variety of species and were coll. from Scotland and Oregon, U.S.A.

GU-255. Scotland

\begin{tabular}{ccc}
$\delta \mathrm{C}^{14 \%}$ & $\delta \mathrm{C}^{13 \%} \%$ & $\Delta \%$ \\
\hline $18.7 \pm 0.6$ & -24.9 & $18.7 \pm 0.7$
\end{tabular}

Seeds (Tsuga mertensiana) coll. 1960 from SW Scotland $\left(56^{\circ} 30^{\prime}\right.$ $\mathrm{N}$ Lat, $3^{\circ} 30^{\prime} \mathrm{W}$ Long). 
GU-256. Scotland

\begin{tabular}{ccc}
$\delta \mathrm{C}^{14} \%$ & $\delta \mathrm{C}^{13} \%$ & $\Delta \%$ \\
\hline $21.6 \pm 0.6$ & -28.9 & $22.5 \pm 0.6$
\end{tabular}

Seeds (Pinus mugo) coll. 1961 from NW Scotland $\left(57^{\circ} 30^{\prime} \mathrm{N}\right.$ Lat, $4^{\circ} 30^{\prime}$ W Long).

GU-257. Scotland

$$
29.4 \pm 0.7 \quad-26.2 \quad 29.8 \pm 0.7
$$

Seeds (Pinus mugo) coll. 1962 from NW Scotland (57 $30^{\prime}$ N Lat, $4^{\circ} 30^{\prime}$ W Long).

GU-258. Scotland $\quad 85.4 \pm 1.2 \quad-26.8 \quad 86.1 \pm 1.2$ W Long).

Seeds (Pinus sylvestris) coll. 1963 from Moray (57 $30^{\prime} \mathrm{N}$ Lat, $3^{\circ} 30^{\prime}$

GU-259. Scotland

$93.8+1.2 \quad-26.7 \quad 94.4 \pm 1.3$

Seeds (Pinus sylvestris) coll. 1964 from Moray (57 $30^{\prime} \mathrm{N}$ Lat, $3^{\circ} 30^{\prime}$ W Long).

GU-53. Scotland $\quad 72.9 \pm 1.0 \quad-26.9 \quad 73.5 \pm 1.0$

Seeds (Pinus sylvestris) coll. 1965 from Moray (57 $30^{\prime} \mathrm{N}$ Lat, $3^{\circ} 30^{\prime}$ W Long).

GU-260. Scotland $\quad 69.9 \pm 0.8 \quad-29.4 \quad 71.4 \pm 0.8$ Seeds (Pinus sylvestris) coll. 1966 from S Scotland $\left(55^{\circ} 30^{\prime} \mathrm{N}\right.$ Lat, $3^{\circ} 30^{\prime}$ W Long).

$\begin{array}{llll}\text { GU-261. Scotland } & 62.7 \pm 0.7 & -25.7 & 62.9 \pm 0.7\end{array}$

Seeds (Larix decidua) coll. 1967 from Moray (57 $30^{\prime} \mathrm{N}$ Lat, $3^{\circ} 30^{\prime}$ W Long).

GU-262. England $\quad 55.7 \pm 0.7 \quad-33.0 \quad 58.2 \pm 0.8$

Seeds (Fagus sylvatica) coll. 1967 from Cirencester $\left(51^{\circ} 40^{\prime} \mathrm{N}\right.$ Lat, $1^{\circ} 57^{\prime}$ W Long).

GU-263. Scotland $\quad 59.4 \pm 0.7 \quad-29.8 \quad 60.9 \pm 0.7$ Seeds (Picea sitchensis) coll. 1968 from N Scotland $\left(58^{\circ} \mathrm{N}\right.$ Lat, $4^{\circ} 30^{\prime}$ W Long).

GU-264. Oregon $\quad 33.4 \pm 1.0 \quad-28.0 * \quad 34.2 \pm 1.0$ Seeds (Pseudotsuga taxifolia) coll. $1959\left(45^{\circ} \mathrm{N}\right.$ Lat, $120^{\circ} \mathrm{W}$ Long).

$\begin{array}{llll}\text { GU-265. Oregon } & 18.5 \pm 0.5 & -30.6 & 19.8 \pm 0.6\end{array}$ Seeds (Abies grandis) coll. 1960 (45 ${ }^{\circ} \mathrm{N}$ Lat, $120^{\circ} \mathrm{W}$ Long).
GU-266. Oregon
$19.9 \pm 0.6 \quad-26.5$
$20.3 \pm 0.6$

Seeds (Picea sitchensis) coll. 1961 (45 ${ }^{\circ} \mathrm{N}$ Lat, $120^{\circ} \mathrm{W}$ Long).
$\begin{array}{llll}\text { GU-267. Oregon } & 28.7 \pm 0.8 & -25.6 & 28.8 \pm 0.8\end{array}$

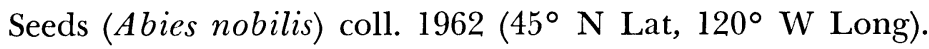
* Estimated, as mass-spectrometric measurements were not available.




\begin{tabular}{ccc}
$\delta \mathrm{C}^{14} \%$ & $\delta \mathrm{C}^{13 \%} \%$ & $\Delta \%$ \\
\hline $78.7 \pm 1.1$ & -26.3 & $79.2 \pm 1.1$
\end{tabular}

Seeds (Pinus contorta) coll 1963 (45 ${ }^{\circ} \mathrm{N}$ Lat, $120^{\circ} \mathrm{W}$ Long).

GU-269. Oregon $\quad 89.2 \pm 1.2 \quad-29.6 \quad 90.9 \pm 1.2$

Seeds (Pinus contorta) coll. 1964 (45 ${ }^{\circ} \mathrm{N}$ Lat, $120^{\circ} \mathrm{W}$ Long).

GU.270. Oregon $\quad 67.6 \pm 1.0 \quad-23.9 \quad 67.2 \pm 1.1$

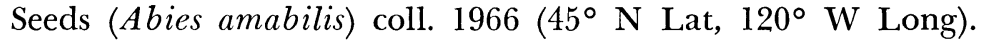

GU-271. Oregon $\quad 70.3 \pm 1.0 \quad-26.1 \quad 70.7 \pm 1.1$

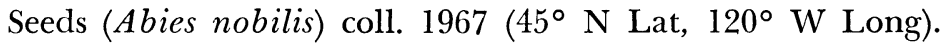

$\begin{array}{llll}\text { GU-272. Oregon } & 58.7 \pm 0.7 & -28.4 & 59.8 \pm 0.8\end{array}$

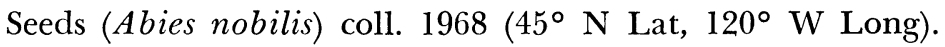

Comment: $\mathrm{C}^{14}$ activities of $\mathrm{N}$ hemispheric tree seeds accurately reflect atmospheric levels during seed growth periods. Rate of equilibration of atmospheric $\mathrm{C}^{14}$ concentrations since 1963 is approximated by the exoression $\Delta_{\mathrm{t}}=97 \mathrm{e}^{-0.10 \mathrm{t}}$ where $\Delta_{\mathrm{t}}$ is the tropospheric $\mathrm{C}^{14}$ concentration $\%$ ) at time and yr after 1963. Discrepancies between the 2 seed series (Scotland and Oregon) although in part statistical, may also be due to slightly different growth periods and to minor disequilibrium in atmospheric $\mathrm{C}^{14}$ distribution in $\mathrm{N}$ Lats.

IV. FLAX SEEDS, CEREAL, AND WOOL SAMPLES

A variety of biospheric materials including flax seeds, and cereals coll. near Belfast, N Ireland (54 $35^{\prime} \mathrm{N}$ Lat, $5^{\circ} 50^{\prime} \mathrm{W}$ Long) and English wool samples of known age were analyzed to permit estimation of past atmospheric $\mathrm{C}^{14}$ activities. Samples were provided by the $\mathrm{N}$ Ireland Ministry of Agriculture.

GU-273. Flax seeds

\begin{tabular}{ccc}
$\delta \mathrm{C}^{14} \%$ & $\delta \mathrm{C}^{13 \%} \%$ & $\Delta \%$ \\
\hline$-3.5 \pm 0.6$ & -33.2 & $-1.9 \pm 0.6$
\end{tabular}

Seeds (Linum usitatissimum) coll. 1934.

GU-274. Oats

$$
-2.4 \pm 0.5 \quad-30.3 \quad-1.3 \pm 0.5
$$

Seeds (Avena sterilis) coll. 1935.

GU-275. Barley

$$
-4.0 \pm 0.6
$$

$-3.0 \pm 0.6$

Seeds (Hordeum distichum) coll. 1936.

GU-276. Flax seeds

$-4.3 \pm 0.5$

$-29.9$

$-3.4 \pm 0.5$

Seeds (Linum usitatissimum) coll. 1936.

GU-277. Flax seeds $-4.7 \pm 0.7$ $-32.6 \quad-3.2 \pm 0.7$

Seeds (Linum usitatissimum) coll. 1938. 
GU-278. Flax seeds

\begin{tabular}{ccc}
$\delta \mathrm{C}^{14} \%$ & $\delta \mathrm{C}^{13 \%} \%$ & $\Delta \%$ \\
\hline$-4.5 \pm 0.5$ & -30.7 & $-3.4 \pm 0.5$
\end{tabular}

Seeds (Linum usitatissimum) coll. 1938.

GU-279. Flax seeds $\quad-3.8 \pm 0.5 \quad-30.1 \quad-2.8 \pm 0.5$

Seeds (Linum usitatissimum) coll. 1940.

GU-280. Flax seeds $\quad-3.8 \pm 0.6 \quad-32.1 \quad-2.5 \pm 0.6$

Seeds (Linum usitatissimum) coll. 1942.

GU-281. Flax $\quad-4.4 \pm 0.5 \quad-30.7 \quad-3.4 \pm 0.6$

Straw (Linum usitatissimum) coll. 1943.

GU-282. Flax seeds $\quad-5.3 \pm 0.6 \quad-32.6 \quad-3.8+0.6$

Seeds (Linum usitatissimum) coll. 1944.

GU-283. Flax seeds $\quad-5.8 \pm 0.5 \quad-30.7 \quad-4.7 \pm 0.5$

Seeds (Linum usitatissimum) coll. 1945.

GU-284. Flax seeds $\quad-6.1 \pm 0.5 \quad-32.0 \quad-4.8 \pm 0.6$

Seeds (Linum usitatissimum) coll. 1946.

GU-285. Flax $\quad-6.0 \pm 0.4 \quad-30.7 \quad-4.9 \pm 0.4$

Straw (Linum usitatissimum) coll. 1947.

GU-286. Flax seeds $\quad-6.1 \pm 0.6 \quad-31.0 \quad-5.0 \pm 0.6$

Seeds (Linum usitatissimum) coll. 1948.

GU-287. Flax seeds $\quad-5.1 \pm 0.6 \quad-29.1 \quad-4.3 \pm 0.6$ Seeds (Linum usitatissimum) coll. 1950.

GU-288. Wool, $1962 \quad 20.4 \pm 0.6 \quad-30.7 \quad 21.7 \pm 0.6$

Comment: wool sample has $\mathrm{C}^{11}$ content representative of 1961 atmospheric $\mathrm{C}^{14}$ levels.

GU-289. Wool $\quad-3.0 \pm 0.8 \quad-31.8 \quad-1.7 \pm 0.8$

Wool coll. 1851 from NE England (54 $\mathrm{N}$ Lat, $1^{\circ} \mathrm{W}$ Long).

GU-290. Wool $\quad-2.3 \pm 0.7 \quad-27.5 \quad-1.8 \pm 0.8$

Wool coll. 1851 from NE England (54 ${ }^{\circ} \mathrm{N}$ Lat, $1^{\circ} \mathrm{W}$ Long).

GU-291. Wool $\quad-4.1 \pm 0.5 \quad-30.5 \quad-3.0 \pm 0.6$

Wool coll. 1944 from NE England (54 $4^{\circ} \mathrm{N}$ Lat, $1^{\circ} \mathrm{W}$ Long).

Comment: results of analyses of malt whiskies, vintage wines, flax seeds, and other biospheric materials indicate that $\mathrm{N}$ hemisphere $\mathrm{C}^{14}$ activities have fluctuated significantly on an annual basis during the time period 1890-1950. The causes of these variations are to be discussed elsewhere. 


\section{Mortar series}

GU-292. Carlisle Castle mortar

$1158 \pm \mathbf{5 7}$

Mortar from “De Ireby's Tower" Carlisle Castle $\left(54^{\circ} 47^{\prime} \mathrm{N}\right.$ Lat, $2^{\circ} 55^{\prime} \mathrm{W}$ Long), from ground floor W room, Garderobe entrance, 9.5 $\mathrm{ft}$ from ground level, $2 \mathrm{ft}$ from interior wall face, and $1.5 \mathrm{ft}$ above lower side of stone lintel. Coll. and subm. 1967 by Ministry of Public Bldgs. and Works, Ancient Monuments Branch. Comment: true age is 580 . Sample prepared from 1st $\mathrm{CO}_{2}$ fraction during acid hydrolysis and contains less contaminant old carbon than GU-66 (2002 \pm 58$)$ prepared from the total $\mathrm{CO}_{2}$ yield (Radiocarbon, 1969, v. 11, p. 51).

\section{GU-293. Carlisle Castle mortar}

$2936 \pm 72$

986 в.C.

Same mortar sample as GU-292 (above) and GU-66 but prepared from the 2nd $\mathrm{CO}_{2}$ fraction during hydrolysis. Comment: discrepancies between 1st, 2nd, and over-all fractions not due to fractionation since mass spectrometric measurements performed. Presumably non-crystalline carbonate (from atmospheric $\mathrm{CO}_{2}$ ) is hydrolyzed preferentially to the carbonate of calcareous sands and/or limestone residues.

GU-294.

$738 \pm 52$

Mortar from Projecting Garderobe Bay Hampton Court Palace $\left(51^{\circ}\right.$ $25^{\prime} \mathrm{N}$ Lat, $0^{\circ} 24^{\prime} \mathrm{W}$ Long), from top of wall immediately below courtyard paving cobbles. Coll. and subm. 1967 by Ministry of Public Bldgs. and Works, Ancient Monuments Branch. Comment: true age is 440 . Contamination by old carbon evident.

\section{GU-295. London Tower mortar}

\begin{tabular}{ccc}
$\delta \mathrm{C}^{14 \%} \%$ & $\delta \mathrm{C}^{13 \%} \%$ & $\Delta \%$ \\
\hline $7.09 \pm 0.59$ & -17.09 & $5.39 \pm 0.61$
\end{tabular}

Mortar from Cold Harbour Tower, Tower of London $\left(51^{\circ} 32^{\prime} \mathrm{N}\right.$ Lat, $0^{\circ} 05^{\prime} \mathrm{W}$ Long), from NW drum of tower immediately above footing offset and present ground level. Repair mortar from 1953. Coll. and subm. 1967 by Ministry of Public Bldgs. and Works, Ancient Monuments Branch. Comment: $\Delta$ value representative of post-1953 nuclear era with same $\mathrm{C}^{1.4}$ content as 1956 atmosphere. Thus mortar "hardening" appears to have reached an advanced stage during the 1 st 5 to $10 \mathrm{yr}$ (since significant incorporation of 1963 atmospheric $\mathrm{C}^{14}$ would have been readily detectable).

\section{GU-296. Orford Castle mortar}

Mortar from W Tower Orford Castle $\left(52^{\circ} 05^{\prime} \mathrm{N}\right.$ Lat, $1^{\circ} 35^{\prime}$. Long), from $\mathrm{W}$ wall of tower $35 \mathrm{ft}$ above $\left(525^{\circ} \mathrm{N}\right.$ Lat, $1^{\circ} 35^{\prime} \mathrm{W}$ 1968 by Ministry of Public Bldgs. and Works, Ancient Monuments 
Branch. Comment: true age is 800 . Sample appears contaminated to $>50 \%$ by inactive carbon.

\section{GU-297. Conway Town Wall mortar}

Mortar from Conway Town Walls ( $53^{\circ} 17^{\prime} \mathrm{N}$ Lat, $3^{\circ} 50^{\prime} \mathrm{W}$ Long), from steps outside $\mathrm{E}$ tower $65 \mathrm{ft}$ from end of tower and from $0.5 \mathrm{ft}$ to $1.5 \mathrm{ft}$ into wall. Coll. and subm. 1968 by Ministry of Public Bldgs. and Works, Ancient Monuments Branch. Comment: true age is 680 and thus contamination by old carbon is evident.

\section{GU-298. Hampton Court mortar}

$370 \pm 31$

Mortar from Apt 35, Wolsey Rooms Hampton Court Palace $\left(51^{\circ}\right.$ $25^{\prime} \mathrm{N}$ Lat, $0^{\circ} 24^{\prime} \mathrm{W}$ Long), from brickwork on internal wall ground floor. Coll. and subm. by Ministry of Public Bldgs. and Works, Ancient Monuments Branch. Comment: true age is ca. 420 and thus contamination by old carbon is not present.

General Comment: this series of data from mortar samples confirms inherent unreliability of this material for dating purposes in the U.K. (Baxter and Walton, 1970). Studies by Stuiver and Smith (1965) and Delibrias and Labeyrie (1965) suggest that conflicting opinions exist on the value of mortar for dating.

\section{GU-299. Kilphedir hut circles, Sutherland, Scotland}

$2370 \pm 40$

Charcoal (birch and hazel) from Hut Circle III, 1 of 5 in locality just below turf at Kilphedir site, Sutherland, Scotland, $3.5 \mathrm{mi}$ from sea at Helmsdale $\left(58^{\circ} 09^{\prime} \mathrm{N}\right.$ Lat, $3^{\circ} 43^{\prime} \mathrm{W}$ Long), $400 \mathrm{ft}$. Coll. and subm. 1968 by H. Fairhurst, Archaeol. Dept., Univ. of Glasgow. Comment: age (based on assumption of $\delta \mathrm{C}^{13}=-28.10 \%$, is in reasonable agreement with archaeologic assessment of ca. 300 B.c.

\section{REFERENCES}

Baxter, M. S. and Walton, A., 1970, Radiocarbon dating of mortars: Nature, v. 225,

p. 937.
Baxter, M. S., Ergin, M., and Walton, A., 1969, Glasgow University radiocarbon measurements I: Radiocarbon, v. 11, p. 43-52.

Delibrias, G. and Labeyrie, J., 1965, The dating of mortars by the carbon-14 method: 6 th internatl. conf. on radiocarbon and tritium dating proc., Pullman, Washington, June 1965 , p. 344-347.

L'Orange, R. and Zimen, K., 1968, C-14 Aus kernwassen-explosionen im carbonat und im kollagen men schlicher knochen: Naturwissenschaften, v. 55, p. 492.

Stuiver, M. and Smith, C. S., 1965, Radiocarbon dating of ancient mortar and plaster: 6 th internatl. conf. on radiocarbon and tritium dating proc., Pullman, Washington, June 1965, p. 338-343. 